\title{
Vector Rogue Waves in a Carbon Nanotube Mode-Locked Fiber Laser
}

\author{
Hani Kbashi, Mohamed Al-Araimi, Alex Rozhin, Sergey V. Sergeyev \\ School of Engineering and Applied Science, Aston University, Aston Triangle, Birmingham B4 7ET, UK
}

Mechanisms of rogue waves (RWs) emergence have been extensively studied in fiber lasers with nonlinearly driven cavities [1], Raman fiber amplifiers and lasers [2], and fiber lasers via modulation of the pump [3]. Previously, it has been found that RWs can be emerged because of soliton-soliton interaction through the overlapping of their tails or soliton-dispersive wave interaction. The result of these interactions is a coupling enhancement that leads to chaotic pulse bunching in the form of soliton rain at the time scale of a round-trip time in a high pump power $(800 \mathrm{~mW})$ [4]. Such interaction can be controlled by the pump power modulation or/and by injecting a weak seeding signal and noise. Here, we report for the first time the observation of the soliton rain and soliton-soliton interaction that leads to the optical rogue wave emergence in a carbon nano-tube saturable absorber mode-locked fiber laser at low pump power $(140 \mathrm{~mW})$ without pump modulation or injecting a weak signal and noise to the laser cavity. As we shown recently [5] that by tuning the interaction of two orthogonalstates of polarization (SOP) of the in-cavity birefringent and also the pump SOP whereas the polarization instability leading to the emergence of different optical RW events.

The experiment setup is illustrated in Fig. 1. A fiber-pigtailed $980 \mathrm{~nm}$ laser diode with a maximum pumping power of $300 \mathrm{~mW}$ was used to pump the $75 \mathrm{~cm}$ long segment of Er-80 gain medium. All the other fibers used (17 $\mathrm{m}$ in total) were standard single-mode fibers (SM-28). We observed that, the dynamics and flow of solitons can be adjusted with tuning the intra-cavity polarization controller, and can also be adjusted with the pump power. With such flexibility, numerous dynamical regimes become accessible besides standard mode locking, by simply altering the orientation of the polarization controllers, among which the soliton rain dynamics have been observed in the oscilloscope traces as illustrated in Fig. 2 a and d for $140 \mathrm{~mW}$ and $240 \mathrm{~mW}$ correspondingly. Probability distribution function (PDF) for both of the these regimes yield a long tail shape which is far from the normal Gaussian distribution as shown in Fig. $2 \mathrm{~b}$ and e. We confirmed that the RW events emerged from the soliton-soliton interaction by analyzing the collecting data using the spatio-temporal map as it illustrated in Fig. $2 \mathrm{c}$ and $\mathrm{f}$ for $140 \mathrm{~mW}$ and $240 \mathrm{~mW}$ respectively.

In summary, we have experimentally observed the vector nature of rogue wave patterns in the passively modelocked fiber laser based on a carbon nanotube saturable absorber. These patterns were sensitive to the pump power and the orientations of the intra-cavity PCs.
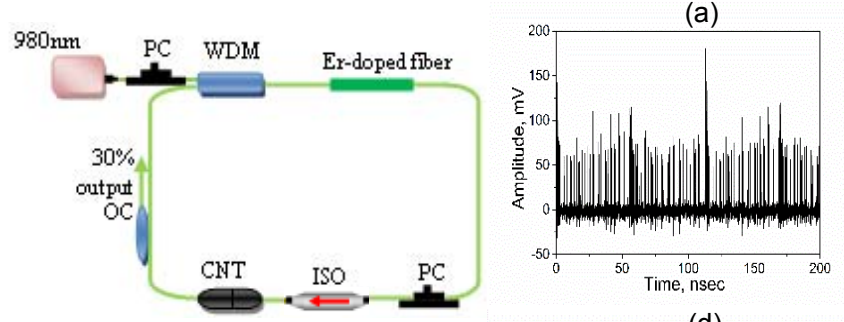

(d)

Fig. 1: The Experimental setup. WDM: wavelength Division multiplexing;

PC: polarization controller; ISO: optical isolator; CNT: carbon nanotube SA; and OC: output coupler
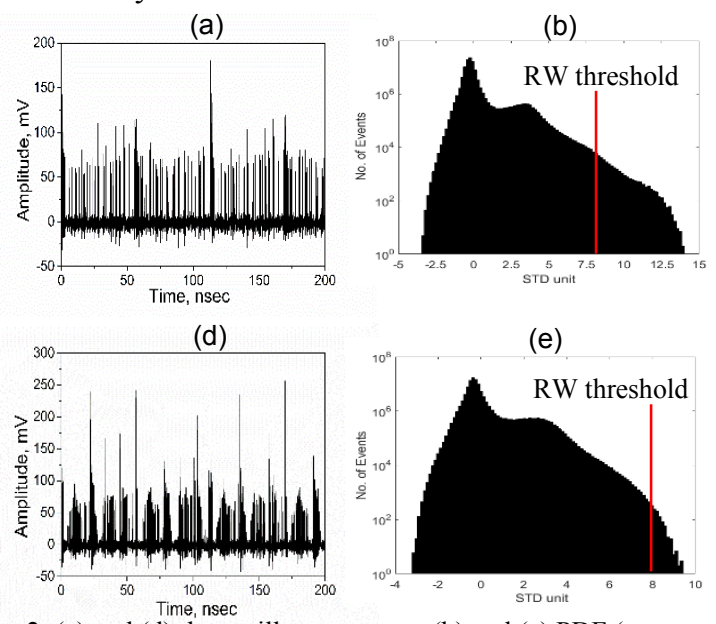

(e)

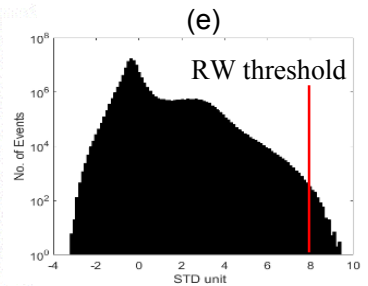

(c)

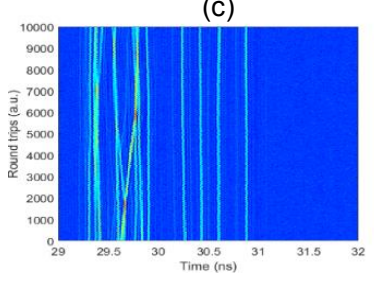

(f)

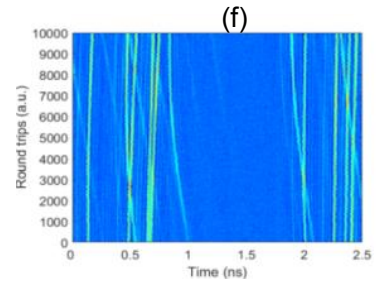

Fig. 2: (a) and (d) the oscilloscope trace, (b) and (e) PDF (output power is normalized to the standard deviation, STU; (c) and (f) the pulse-pulse interaction in the spatio-temporal map at $140 \mathrm{~mW}$ and $240 \mathrm{~mW}$ of the pump power respectively

\section{References}

[1]. D. R. Solli, C. Ropers, P. Koonath, and B. Jalali, “Optical rogue waves,” Nature 450, 1054-1057 (2007).

[2]. K. Hammani, C. Finot, J. M. Dudley, and G. Millot, "Optical rogue-wavelike extreme value fluctuations in fiber Raman amplifiers," Opt. Express 16, 16467-16474 (2008).

[3]. A. N. Pisarchik, R. Jaimes-Reategui, R. Sevilla-Escoboza, G. Huerta-Cuellar, and M. Taki, "Rogue waves in a multistable system," Phys. Rev. Lett. 107, 274101 (2011).

[4]. S. Chouli and Ph. Grelu, "Soliton rains in a fiber laser: An experimental study," Phys. Rev. A, 81, 063829 (2010).

[5]. S. Kolpakov, H. Kbashi, S. Sergeyev, "Dynamics of vector rogue waves in a fiber laser with a ring cavity," Optica, 3, 8, 870 (2016). 